\title{
Descompressão orbitária antro-etmoidal na orbitopatia distireoidiana
}

\author{
Antro-ethmoidalorbitaldecompression in dysthyroid orbitopathy
}

\author{
Mário Luiz Ribeiro Monteiro' \\ Miriam Rotenberg' Ostroscki ${ }^{2}$ \\ André Luis Borba da Silva² \\ Walter Bloise $^{3}$
}

\begin{tabular}{l} 
RESUMO \\
\hline Objetivo: Avaliar os resultados e as complicações da descompressão \\
orbitária antro-etmoidal em pacientes com orbitopatia distireoidiana. \\
Métodos: 14 pacientes sendo 10 do sexo feminino, com média de idade \\
de 41,7 anos, foram submetidos a 22 cirurgias de descompressão \\
orbitária. Em 3 cirurgias (2 pacientes), na fase ativa da oftalmopatia a \\
indicação cirúrgica foi ulceração de córnea, com risco de perfuração \\
corneana. Estes pacientes estavam recebendo corticosteróides associa- \\
dos a ciclofosfamida e radioterapia tendo o tratamento prosseguido \\
após a cirurgia. Dezenove órbitas foram operadas em fase inativa da \\
orbitopatia por indicação cosmética associada a desconforto ocular \\
por exposição corneana. Resultados: A redução da proptose oscilou \\
entre 1 e 6 mm (média 3,91 mm). Nos pacientes operados na fase aguda \\
a redução média foi de $5,33 \pm 0,27$ mm e na fase sequelar foi de $3,68 \pm$ \\
0,25 mm. Nenhum paciente apresentou diplopia conseqüente a cirurgia. \\
Correção de estrabismo prexistente foi realizada em 2 pacientes e \\
tarsorrafia temporária foi associada a cirurgia descompressiva nas 3 \\
cirurgias realizadas na fase aguda. Seis pacientes foram subseqüente- \\
mente submetidos à correção de retração palpebral prexistente. Em uma \\
paciente houve piora da retração palpebral inferior como conseqüência \\
da descompressão orbitária. Houve diminuição transitória da sen- \\
sibilidade na região malar em quase todos os pacientes mas em nenhum \\
deles ela foi definitiva. Conclusões: A descompressão óssea antro- \\
etmoidal da órbita é eficiente em reduzir a proptose em pacientes com \\
orbitopatia distireoidiana e apresenta baixa incidência de complica- \\
ções. Associada a outras modalidades terapêuticas pode ser um pro- \\
cedimento útil em casos graves na fase congestiva. Da mesma forma, \\
se mostrou benéfica na reabilitação cosmética de indivíduos na fase \\
seqüelar.
\end{tabular}

Descritores: Doenças orbitárias/terapia; Descompressão; Exoftalmia/cirurgia; Doença de Graves/terapia
Trabalho apresentado no XIII Congresso Brasileiro de Prevenção da Cegueira e Reabilitação Visual, de 7 a 10 de setembro de 1998, no Rio de Janeiro (RJ) e no VIII Congresso da Sociedade Latinoamericana de Tireóide, de 27 a 30 de maio de 1999, em Foz do Iguaçu (PR).

${ }^{1}$ Prof. Livre Docente, Divisão de Clínica Oftalmológica do Hospital das Clínicas da Faculdade de Medicina da Universidade de São Paulo.

2 Pós graduando da Faculdade de Medicina da Universidade de São Paulo.

${ }^{3}$ Prof. Livre Docente, Departamento de Endocrinologia do Hospital das Clínicas da Faculdade de Medicina da Universidade de São Paulo.

Endereço para correspondência: Mário L. R. Monteiro, Av. Angélica, 1757 - conj. 61 - São Paulo (SP) CEP 01227-200.

\section{INTRODUÇ̃̃̃O}

A orbitopatia distireoidiana é uma das afecções mais freqüentes da órbita e pode levar a inúmeras alterações funcionais e estéticas tais como: proptose, retração palpebral, distúrbios da motilidade ocular extrínseca, alterações congestivas nas pálpebras e conjuntiva, exposição corneana e ocasionalmente neuropatia óptica compressiva ${ }^{(1)}$.

Além do tratamento clínico e da radioterapia, vários procedimentos cirúrgicos podem ser necessários no tratamento dessa afecção. A descom- 
pressão orbitária é um desses procedimentos e pode ser realizada na fase aguda da doença, para tratamento da perda visual decorrente de lesão corneana ou do nervo óptico, ou na fase crônica para corrigir a deformidade estética decorrente da proptose $^{(2-3)}$. Esse procedimento pode ser feito por diversas vias de acesso: lateral, anterior, inferior, superior, medial ou por associações destas ${ }^{(1)}$. A maioria das técnicas utilizadas atualmente combina a remoção de duas ou mais paredes orbitárias. A remoção das paredes inferior e medial pode ser feita por via transantral, transconjuntival ou palpebral ${ }^{(4-6)}$. Outros autores combinaram a remoção das paredes medial e inferior com a lateral ${ }^{(7)}$, ou utilizaram a remoção de parte das 4 paredes orbitárias para correção de grandes exoftalmias ${ }^{(8)}$.

As diversas técnicas de descompressão óssea da órbita têm sido consideradas como procedimentos cirúrgicos agressivos com muitas complicações possíveis, em especial os desequilíbrios da motilidade ocular extrínseca. No entanto, muitas das complicações são decorrentes de cirurgias extensas para obter uma correção muito grande da proptose. Além disso, no passado a cirurgia era realizada quase que exclusivamente para tratar quadros muito severos de pacientes com perda visual por comprometimento do nervo óptico. Nesses indivíduos o comprometimento da motilidade ocular geralmente já existia antes da descompressão orbitária.

O objetivo deste trabalho é relatar nossa experiência com a descompressão orbitária, usando uma abordagem relativamente conservadora, com remoção de parte das paredes inferior e medial da órbita, que pode ser feita por via conjuntival ou palpebral ${ }^{(5-6)}$. Nossa casuística inclui predominantemente pacientes com deformidade estética e mostra que tal procedimento pode ser bastante útil para reduzir a proptose e é associado a uma incidência muito baixa de complicações.

\section{MÉTODOS}

No período de janeiro de 1992 a março de 1998, 14 pacientes foram submetidos a descompressão orbitária antro-etmoidal. A cirurgia foi bilateral em 8 indivíduos e unilateral em 6 . Ao todo, 22 cirurgias foram realizadas. Todos pacientes tinham diagnóstico clínico e laboratorial de doença de Graves e haviam sido submetidos a tratamento clínico medicamentoso e/ou iodo radioativo. Dois pacientes (3 cirurgias) foram operados na fase aguda (congestiva) da doença, em decorrência de risco eminente de perfuração corneana, a despeito do uso de ciclofosfamida e de altas doses de corticosteróides. Os demais foram operados na fase não inflamatória, já sob controle adequado, na maior parte das vezes por razões estéticas, embora a presença de irritação conjuntival crônica fosse um fator coadjuvante. Dois apresentavam hipotropia na posição primária do olhar.

A orbitopatia era bilateral em 12 casos e unilateral em 2. A cirurgia foi unilateral em 6 pacientes. Em 4, embora a proptose fosse bilateral, julgou-se necessária a descompressão apenas de uma órbita. Em apenas 2 pacientes a cirurgia foi bilateral e simultânea.

A cirurgia foi realizada segundo técnica descrita por McCord $^{(5)} \mathrm{em} 12$ pacientes. Sob anestesia geral e após infiltração de xilocaína com adrenalina realiza-se uma cantotomia lateral e lise do ligamento cantal lateral inferior. A pálpebra inferior liberada é tracionada com fio de seda 4-0 e a conjuntiva incisada 6 a $8 \mathrm{~mm}$ abaixo do tarso, do canto lateral para o medial até a região da carúncula. Utiliza-se retrator maleável para deslocar o globo ocular para cima e afastadores são colocados na incisão conjuntival inferior, de tal forma a esticar os retratores da pálpebra sobre o rebordo orbitário. A fáscia capsulopalpebral é aberta expondo-se o periósteo. Move-se o retrator maleável de tal forma a afastar também o músculo oblíquo inferior e protegê-lo juntamente com o globo ocular. O periósteo é incisado ao longo de toda a extensão do rebordo orbitário. Afasta-se o periósteo descolado com retrator e disseca-se todo o assoalho orbitário com descolador de periósteo e identifica-se o nervo infra-orbitário. Realiza-se uma abertura na parede inferior da órbita de preferência no seu setor mais medial, com osteótomo, criando-se uma comunicação com o seio maxilar. Remove-se toda a parede inferior na porção medial ao nervo infra-orbitário, até a parede posterior do seio maxilar. A parede inferior lateral ao nervo infra-orbitário não foi removida em nossos pacientes. Removemos também a parede medial abaixo do forame das artérias etmoidais anterior e posterior. Após completada a remoção óssea e controlado o sangramento da mucosa sinusal, procede-se à incisão da periórbita (duas ou mais) no plano sagital, idealmente colocadas entre os músculos retos. Incisões transversais também são realizadas de tal forma que se observe herniação da gordura para os seios maxilar e etmoidal. A conjuntiva é suturada com fio absorvível 5-0 ou 6-0 e o tendão cantal lateral é suturado ao periósteo com fio inabsorvível 5-0. A pele é suturada com fio de nylon 6-0 $0^{(1,5)}$.

Em dois pacientes (casos 5 e 6) a incisão foi realizada através da pálpebra inferior até atingir o periósteo, sendo os passos seguintes semelhantes ao que foi acima descrito ${ }^{(6)}$.

\section{RESULTADOS}

A tabela 1 mostra a idade, sexo, indicação cirúrgica e lado dos pacientes operados. Quatro eram do sexo masculino e 10 do feminino. A idade dos pacientes variou entre 15 e 71 anos (média 41,7 anos). Dois pacientes (casos 1 e 13) foram operados na fase aguda da doença, apresentando ulceração de córnea com piora progressiva a despeito do tratamento clínico com corticóide e ciclofosfamida (casos 1 e 13) e radioterápico (caso 1), além do tratamento tópico com colírios e pomadas. Todos os demais tiveram a indicação primariamente estética embora em vários deles a proptose importante também ocasionasse desconforto ocular com tipo ardor e sensação de corpo estranho devido à exposição corneana, a despeito do uso de 


\begin{tabular}{|c|c|c|c|c|c|}
\hline CASO & IDADE & SEXO & LADO OPERADO & INDICAÇÃO & SITUAÇÃO ENDÓCRINA \\
\hline 1 & 55 & Masculino & Ambos & Úlcera de córnea & Graves Agudo \\
\hline 2 & 27 & Feminino & Esquerdo & Estética & Seqüela \\
\hline 3 & 62 & Feminino & Esquerdo & Estética & Seqüela \\
\hline 4 & 15 & Feminino & Ambos & Estética & Seqüela \\
\hline 5 & 71 & Feminino & Ambos & Estética & Seqüela \\
\hline 6 & 41 & Masculino & Esquerdo & $\begin{array}{l}\text { Estética, Estrabismo, } \\
\text { Exposição de córnea }\end{array}$ & Seqüela \\
\hline 7 & 31 & Feminino & Ambos & Estética & Seqüela \\
\hline 8 & 39 & Feminino & Ambos & Estética & Seqüela \\
\hline 9 & 46 & Feminino & Ambos & Estética & Seqüela \\
\hline 10 & 20 & Masculino & Ambos & Estética & Seqüela \\
\hline 11 & 31 & Feminino & Ambos & Estética & Seqüela \\
\hline 12 & 52 & Feminino & Direito & $\begin{array}{l}\text { Estética, Estrabismo, } \\
\text { Exposição de córnea }\end{array}$ & Seqüela \\
\hline 13 & 39 & Masculino & Direito & Úlcera de córnea & Graves Agudo \\
\hline 14 & 48 & Feminino & Esquerdo & Estética & Seqüela \\
\hline
\end{tabular}

colírios lubrificantes. Em dois pacientes (casos 6 e 12) havia também estrabismo vertical restritivo associado à proptose e a descompressão foi indicada como tratamento preliminar antes da correção do estrabismo.

A tabela 2 mostra as exoftalmometrias pré e pós-operatórias, a redução da proptose obtida, as complicações e os outros procedimentos cirúrgicos necessários após as 22 cirurgias de descompressão orbitária realizadas nesta série.

A correção da proptose variou entre 1 e $6 \mathrm{~mm}$, com média de $3,91 \pm 0,25 \mathrm{~mm}$. Os 2 pacientes com quadro agudo congesti- vo apresentaram melhora importante da ulceração corneana embora outras medidas clínicas como uso de corticóide, ciclofosfamida e radioterapia tenham sido empregadas. Em um dos pacientes (caso 1) apenas um olho apresentava ulceração corneana com risco eminente de perfuração e foi operado após tentativa clínica com corticóides e ciclofosfamida e após ter sido iniciado o tratamento radioterápico. Quanto ao olho contralateral, menos afetado, aguardamos vários meses com uso de corticóide e ciclofosfamida e realização de duas sessões de radioterapia (total de 2500 rads). Apesar disso houve agrava-

\begin{tabular}{|c|c|c|c|c|c|}
\hline CASO & EXOF. PRÉ & EXOF. PÓS & REDUÇÃO & COMPLICAÇÕES & OUTROS PROCEDIMENTOS \\
\hline \multirow[t]{2}{*}{1} & OD 27 & OD 22 & 5 & - & Tarsorrafia \\
\hline & OE 28 & OE 22 & 6 & - & Tarsorrafia \\
\hline 2 & OE 23 & OE 18 & 5 & - & Retração palpebral \\
\hline 3 & OE 26 & OE 25 & 1 & Dificuldade remoção óssea & Retração palpebral \\
\hline 4 & $\begin{array}{l}\text { OD } 25 \\
\text { OE } 26\end{array}$ & $\begin{array}{l}\text { OD } 20 \\
\text { OE } 21\end{array}$ & $\begin{array}{l}5 \\
5\end{array}$ & $\begin{array}{l}- \\
-\end{array}$ & $\begin{array}{l}- \\
-\end{array}$ \\
\hline 5 & $\begin{array}{l}\text { OD } 29 \\
\text { OE } 29\end{array}$ & $\begin{array}{l}\text { OD } 25 \\
\text { OE } 25\end{array}$ & $\begin{array}{l}4 \\
4\end{array}$ & $\begin{array}{l}\text { Piora retração inferior } \\
\text { Piora retração inferior }\end{array}$ & $\begin{array}{l}\text { Retração palpebral } \\
\text { Retração palpebral }\end{array}$ \\
\hline 6 & OE 27 & OE 23 & 4 & - & Correção de estrabismo \\
\hline 7 & $\begin{array}{l}\text { OD } 25 \\
\text { OE } 24\end{array}$ & $\begin{array}{l}\text { OD } 20 \\
\text { OE } 21\end{array}$ & $\begin{array}{l}5 \\
3\end{array}$ & $\begin{array}{l}- \\
-\end{array}$ & $\begin{array}{l}- \\
-\end{array}$ \\
\hline 8 & $\begin{array}{l}\text { OD } 27 \\
\text { OE } 26\end{array}$ & $\begin{array}{l}\text { OD } 22 \\
\text { OE } 23\end{array}$ & $\begin{array}{l}5 \\
3\end{array}$ & $\begin{array}{c}\text { Descomp. trans. foria } \\
-\end{array}$ & $\begin{array}{l}\text { Retração palpebral } \\
\text { Retração palpebral }\end{array}$ \\
\hline 9 & $\begin{array}{l}\text { OD } 23 \\
\text { OE } 23\end{array}$ & $\begin{array}{l}\text { OD } 21 \\
\text { OE } 20\end{array}$ & $\begin{array}{l}2 \\
3\end{array}$ & - & $\begin{array}{l}\text { Retração palpebral } \\
\text { Retração palpebral }\end{array}$ \\
\hline 10 & $\begin{array}{l}\text { OD } 23 \\
\text { OD } 23\end{array}$ & $\begin{array}{l}\text { OD } 19 \\
\text { OD } 19\end{array}$ & $\begin{array}{l}4 \\
4\end{array}$ & $\begin{array}{l}- \\
-\end{array}$ & $\begin{array}{l}- \\
-\end{array}$ \\
\hline 11 & $\begin{array}{l}\text { OD } 23 \\
\text { OE } 23\end{array}$ & $\begin{array}{l}\text { OD } 20 \\
\text { OE } 20\end{array}$ & $\begin{array}{l}3 \\
3\end{array}$ & - & $\begin{array}{l}\text { Retração palpebral } \\
\text { Retração palpebral }\end{array}$ \\
\hline 12 & OD 25 & OD 21 & 4 & - & Correção de estrabismo \\
\hline 13 & OD 28 & OD 23 & 5 & - & Tarsorrafia \\
\hline 14 & OE 28 & OE 25 & 3 & - & - \\
\hline
\end{tabular}


mento da exposição corneana e só após a descompressão orbitária obtivemos o controle total do processo. As 3 cirurgias descompressivas indicadas para tratamento de úlcera de córnea foram acompanhadas de tarsorrafia temporária, que foi mantida por 2 a 3 semanas. Nessas cirurgias (casos 1 e 13) a redução média da proptose foi de 5,33 $\pm 0,27 \mathrm{~mm}$.

Os 12 pacientes ( 19 cirurgias) com indicação estética apresentaram melhora importante da proptose. Apesar disso, grande parte dos pacientes necessitaram de correção da retração palpebral para se atingir um efeito estético desejado (Tabela 2). A redução média da proptose nesses pacientes foi de $3,68 \pm 0,25 \mathrm{~mm}$.

Quanto às complicações, pudemos observar apenas perda transitória da sensibilidade na região do nervo infraorbitário. No entanto, nenhum paciente apresentou perda da sensibilidade definitiva, tendo o sintoma desaparecido após algumas semanas. Em uma paciente (caso 8), portadora de exotropia intermitente antiga, houve descompensação transitória da mesma.

Nenhum paciente, desenvolveu diplopia na posição primária do olhar ou na posição de leitura que necessitasse de cirurgia. Os casos 6 e 12, que já apresentavam estrabismo préoperatório, necessitaram correção do estrabismo após a descompressão. Um deles (caso 6) apresentou discreta piora e a outra (caso 12) discreta melhora do desvio com a cirurgia descompressiva. Outro paciente (caso 11), operado na fase aguda da doença e com comprometimento severo da motilidade ocular além de ulceração corneana, ainda se encontra em acompanhamento e poderá necessitar cirurgia corretiva do estrabismo.

Em uma paciente (caso 3) a cirurgia foi difícil devido a parede óssea orbitária ser muito grossa. A remoção foi prejudicada e nesta paciente obtivemos uma correção muito pequena da proptose. Esta cirurgia foi uma das primeiras por nós realizada e acreditamos que com maior experiência o problema poderia ter sido contornado. Outra paciente (caso 5), cuja cirurgia foi feita através de incisão palpebral, apresentou piora da retração inferior em ambos os olhos, embora esta já fosse importante no pré-operatório.

\section{DISCUSSÃO}

Nossos achados revelam que a cirurgia de descompressão óssea da órbita com remoção de apenas parte de 2 paredes orbitárias mostra-se eficiente para reduzir a proptose e é associada a um número baixo de complicações. O tratamento da orbitopatia distireoidiana é bastante complexo dada à multiplicidade de acometimentos e a heterogeneidade das manifestações que acarreta. As dificuldades muitas vezes se iniciam na realização do diagnóstico adequado e se mantém nas demais fases da doença. A fase inicial da afecção, caracteriza-se por acometimento inflamatório da órbita que posteriormente é substituído por fibrose e cicatrização dos tecidos orbitários. Muitas vezes a doença segue um curso relativamente brando e autolimitado. No entanto quando o acometimento inflamatório é importante e associado ao comprometimento da musculatura extraocular, e a proptose é progressiva o tratamento deve ser instituído, e pode ser feito com corticosteróides e com radioterapia em doses anti-inflamatórias (geralmente 2000 rads). Em casos de gravidade mais acentuada, especialmente quando existe comprometimento do nervo óptico pode-se utilizar imunossupressão com ciclofosfamida.

Nos casos com orbitopatia congestiva importante, o tratamento primordial é clínico visando o controle do processo. No entanto, quando existe risco eminente de perda visual por ulceração de córnea ou por neuropatia óptica compressiva a descompressão orbitária pode ser útil, como exemplificado em dois dos nossos pacientes. A descompressão, deve portanto, ser utilizada associada ao tratamento clínico nesta fase. Alguns cirurgiões utilizam descompressões extremamente amplas de até quatro paredes em casos de proptose muito acentuada $^{(8)}$. Além disso, até há alguns anos muitos autores utilizavam a descompressão orbitária no tratamento da neuropatia óptica ou exposição corneana importante sem esgotar as tentativas clínicas com corticóide, radioterapia e mesmo imunossupressão. É possível que em casos muito especiais tais procedimentos possam ser necessários. No entanto, observamos que quando associado ao tratamento clínico adequado a cirurgia descompressiva menos agressiva de 2 paredes pode ser bastante útil sendo associada à baixa morbidade, como exemplificado nestes 2 pacientes. A cirurgia na fase aguda deve ser vista, portanto, como um complemento ao tratamento clínico nos casos graves.

É importante salientar que a abordagem por nós utilizada não foi indicada em nenhum paciente com neuropatia óptica compressiva. No mesmo período 3 pacientes com neuropatia óptica grave não responsiva ao tratamento clínico foram submetidos à cirurgia descompressiva utilizando-se outras abordagens, em dois deles descompressão por via transantral e em um a abordagem trans-etmoidal. Acreditamos que nestes casos o objetivo maior é o de descomprimir o ápice da órbita em sua porção medial, uma vez que o músculo reto medial espessado é o principal responsável pela compressão do nervo óptico ${ }^{(9)}$. Embora a descompressão por via transconjuntival possa ser eficaz, acreditamos que uma melhor descompressão do ápice medial da órbita seja conseguido por via trans-antral ou trans-etmoidal.

Inúmeras complicações tem sido descritas como consequentes à descompressão orbitária. Garrity e cols. ${ }^{(2)}$ revisaram 428 casos de orbitopatia de Graves submetidos à descompressão orbitária, por via trans-antral e observaram o aparecimento de diplopia em 64\% de 110 pacientes que não tinham diplopia prévia. Muitos outros pacientes já apresentavam diplopia prévia e ao todo 300 necessitaram cirurgia corretiva para o estrabismo após a descompressão. No entanto, observa-se que em 50,7\% a cirurgia foi indicada por neuropatia óptica, em $27,1 \%$ por orbitopatia congestiva grave e em $8,4 \%$ para reduzir proptose severa antes da cirurgia de estrabismo. Diplopia já 
estava presente em $67 \%$ dos pacientes antes da descompressão. Estes dados indicam que a cirurgia foi realizada em casos de orbitopatia severa, já com comprometimento prévio importante da musculatura extrínseca ocular. Outras complicações relatadas na descompressão orbitária por via trans-antral são: perda da sensibilidade na região do nervo infraorbitário, entrópio, hemorragia e enfisema orbitários, sinusite, lesão do nervo óptico, fístula liquórica e obstrução lacrimal ${ }^{(2,10)}$.

Outras séries utilizando a mesma abordagem trans-antral, relatam uma incidência bem menor de desequilíbrios da motilidade ocular variando entre 14 e $24 \%$ a incidência de cirurgia de estrabismo necessária no pós-operatório ${ }^{(4)}$. Em todas esses trabalhos, observa-se que muitos pacientes já apresentavam desequilíbrios importantes da motilidade ocular que apenas foram acentuados com a cirurgia. Por exemplo, Baylis e cols. em 24 pacientes operados, encontraram 5 (21\%) que necessitaram cirurgia corretiva de estrabismo para tratamento de diplopia no pós-operatório, mas quatro deles já tinham tal sintoma antes da cirurgia ${ }^{(4)}$. Shorr e col. refere que em torno de $30 \%$ dos pacientes já apresentavam alterações da motilidade ocular extrínseca. Por outro lado, este autor refere que pacientes com versões normais no pré-operatório tem incidência muito menor de desequilíbrios da motilidade ocular extrínseca ${ }^{(11)}$.

Estes dados indicam que embora inúmeras complicações possam ocorrer em indivíduos submetidos à descompressão orbitária, elas são muito mais freqüentes quando se utiliza a cirurgia em casos de orbitopatia severa. Muitos desses indivíduos com quadro congestivo grave ou com neuropatia óptica melhoram significativamente quando tratados adequadamente com corticosteróides, radioterapia e drogas imunossupressoras. A descompressão orbitária deve, portanto, ser utilizada como um recurso extremo nos casos agudos, sempre complementando um tratamento clínico adequado.

Nos últimos anos a descompressão orbitária tem sido muito utilizada no tratamento de deformidades estéticas em pacientes com seqüela de orbitopatia distireoidiana ${ }^{(3)}$. Observa-se uma tendência a utilizar cada vez mais abordagens com incisões pelas pálpebras ou pela conjuntiva ${ }^{(12)}$. Cruz e col. relataram sua experiência em 13 pacientes submetidos à descompressão orbitária por via transconjuntival com a remoção de parte das 3 paredes orbitárias e não observaram nenhum caso de desequilíbrio da motilidade ocular extrínseca decorrente da cirurgia. Acreditam que isto se deve à baixa incidência de desequilíbrios da motilidade ocular extrínseca no préoperatório ${ }^{(7)}$.

Enquanto que, na fase aguda, a descompressão orbitária por nós utilizada pode ser vista como um complemento ao tratamento clínico, de maneira análoga, acreditamos que no tratamento da proptose residual causadora de deformidade estética, a cirurgia descompressiva deva ser analisada com parcimônia e associada a outras medidas corretivas, particularmente a correção da retração palpebral e a blefaroplastia. Desta forma, é preferível uma correção menor da proptose, com menor risco de complicações, e através de uma cirurgia menos agressiva, do que uma correção mais agressiva da proptose associada a um maior número de complicações.

Embora Cruz e col. ${ }^{(7)}$ não tenham encontrado complicações significativas com a remoção de 3 paredes por via semelhante àquela por nós utilizado, acreditamos que a remoção da parede lateral da órbita aumenta significativamente a morbidade da cirurgia, e pode levar à deformidade estética, seja pela incisão da pele que é necessária, seja por possível retração dos tecidos acima do arco zigomático removido ${ }^{(13)}$. Observamos que a redução média da proptose por nós obtida foi de $3,91 \mathrm{~mm}$, apenas discretamente inferior a observada por Cruz e cols. $(4,6 \mathrm{~mm})$ com a remoção de 3 paredes $^{(7)}$. Quando associada à correção de eventual retração palpebral e de bolsas palpebrais, na grande maioria das vezes uma correção de 3 a $4 \mathrm{~mm}$ da proptose é suficiente para uma melhora estética acentuada.

\section{CONCLUSÕES}

Em conclusão, em nossa casuística de 22 cirurgias de descompressão óssea antro-etmoidal da órbita em portadores de orbitopatia distireoidiana, observamos melhora significativa da proptose com uma incidência muito baixa de complicações. Embora a cirurgia de descompressão orbitária deva ser considerada com cuidado, nossos resultados mostram que pode ser de grande auxílio como complemento ao tratamento clínico em casos de orbitopatia congestiva severa e no tratamento das deformidades estéticas seqüelares da orbitopatia distireoidiana.

\section{ABSTRACT}

Purpose: To evaluate the results and complications of a restricted (antral-ethmoidal) orbital decompression in the treatment of dysthyroid orbitopathy. Methods: 14 patients (10 female), with a mean age of 41.7 years, were submitted to 22 orbital decompressions. Three surgeries (2 patients) were performed in the congestive phase of the disease due to severe corneal ulceration. These patients were receiving corticosteroids associated with radiotheraphy and cyclophosphamide and continued these treatments after surgery. Nineteen orbits were operated on during the inactive phase of the ophthalmopathy in order to improve ocular discomfort and for cosmetic reasons. Surgeries were performed by a superior approach, using a conjunctival incision in 19 and a palpebral one in 3. Results: Reduction of proptosis ranged from 1 to 6 $\mathrm{mm}$ (average $3.91 \mathrm{~mm}$ ). Mean reduction was $5.33 \pm 0.27 \mathrm{~mm}$ in the patients operated on in the acute phase and $3.68 \pm 0.25 \mathrm{~mm}$ in those operated on in the inactive phase of the ophthalmopathy. None developed diplopia as a result of the surgery. Surgical correction of preexistent strabismus was performed in two patients and temporary blepharorraphy was associated with the decompression in the cases operated on in the acute phase. Six patients were subsequently submitted to surgical 
correction of preexistent lid retraction. In one, inferior lid retraction worsened as a result of the lid incision. Transient loss of sensitivity in the cheek was common but not permanent. Conclusions: Antral-ethmoidal orbital decompression is efficient in reducing proptosis in patients with Graves' ophthalmopathy and is associated with a very low incidence of complications. Combined with other treatment modalities it can be helpful in severe cases in the acute phase and in the cosmetic rehabilitation of patients with residual proptosis.

Keywords: Orbital diseases/therapy; Decompression; Exophthalmos/surgery; Graves' disease/therapy

\section{REFERÊNCIAS}

1. Monteiro MLR, Cruz AAV, Manso PG. Orbitopatia distireoidiana. In: Soares EJC, Moura EM, Gonçalves JOR. Cirurgia plástica ocular. São Paulo: Roca 1997. cap 19.

2. Garrity JA, Fatourechi V, Bergstralh EJ, Bartley GB, Beaty CW, DeSanto LW,
Gorman CA. Results of transantral orbital decompression in 428 patients with severe Graves' ophthalmopathy. Am J Ophthalmol 1993;116:533-47.

3. Lyons CJ, Rootman J. Orbital decompression for disfiguring exophthalmos in thyroid orbitopathy. Ophthalmology 1994;101:223-30.

4. Baylis HI, Call B, Shibata CS. The transantral orbital decompression (Ogura tecnhique) as performed by the ophthalmologist: a series of 24 patients. Ophthalmology 1980;87:1005-12.

5. McCord CD Jr. Orbital decompression for Graves' disease. Exposure through lateral canthal and inferior fornix incision. Ophthalmology 1981;88:533-41.

6. Anderson RL, Linberg JV. Transorbital approach to decompression in Graves' disease. Arch Ophthalmol 1981;99:120-4.

7. Cruz AAV, Guimarães FC, Sakuma C, Mauad A. Descompressão orbital no tratamento da orbitopatia distireoidiana. Arq Bras Oftalmol 1996;59:62-8.

8. Kennerdell JS, Maroon JC. An orbital decompression for severe dysthyroid exophthalmos. Ophthalmology 1982;89:467-72.

9. Ostroscki MR, Monteiro MLR. Neuropatia óptica grave na orbitopatia distireoidiana. Rev Bras Oftalmol. No prelo 2001.

10. Rodrigues Alves CA, Castro DJA da S, Barbosa ICF. Exoftalmo endócrino e descompressão orbitária. Rev Bras Oftalmol 1988;47:41-5.

11. Shorr N, Neuhaus RW, Baylis HI. Ocular motility problems after orbital decompression for dysthyroid ophthalmopathy. Ophthalmology 1982;89:323-8.

12. McCord CD Jr. Current trends in orbital decompression. Ophthalmology 1985;92:21-33.

13. Shore JW, Carvajal J, Westfall CT. Miniplate reconstruction of the lateral orbital rim after orbital decompression for Graves disease. Ophthalmology 1992;99:1433-9.

\title{
XXIII CONGRESSO PAN-AMERICANO DE OFTALMOLOGIA
}

\author{
22 A 25 DE JULHO DE 2001
}

\author{
Hotel Sheraton \\ Buenos Aires - Argentina
}

\begin{abstract}
Informações: Associação Panamericana de Oftalmologia Telf.: (001-817) 265-2831 - Fax: (001-817) 275-3961 e-mail:info@paao.org
\end{abstract}

